\title{
Specific Heat and Entropy of a Three Electron Model in Bismuth Based Cuprate Superconductor
}

\author{
Odhiambo Oloo Jared", Makokha John Wanjala \\ Department of Science Technology and Engineering, Faculty of Science, Kibabii University, Bungoma, Kenya \\ Email address: \\ jodhiambo@kibu.ac.ke (O. O. Jared) \\ *Corresponding author \\ To cite this article: \\ Odhiambo Oloo Jared, Makokha John Wanjala. Specific Heat and Entropy of a Three Electron Model in Bismuth Based Cuprate \\ Superconductor. World Journal of Applied Physics. Vol. 3, No. 2, 2018, pp. 19-24. doi: 10.11648/j.wjap.20180302.11
}

Received: March 27, 2018; Accepted: May 2, 2018; Published: June 11, 2018

\begin{abstract}
A theoretical study considering Bi2201, Bi2212 and Bi2223 bismuth based cuprates whose critical Temperatures $\left(\mathrm{T}_{\mathrm{C}}\right)$ are $20 \mathrm{~K}, 95 \mathrm{~K}$ and $110 \mathrm{~K}$ with one, two and three $\mathrm{CuO}_{2}$ planes respectively; based on a three electron model in Bismuth based cuprates oxide shows that there is a direct correlation between energy of interaction and the number of $\mathrm{CuO}_{2}$ planes at the $\mathrm{T}_{\mathrm{C}}$. The specific heat for a mole of Bismuth based cuprates at $\mathrm{T}_{\mathrm{C}}$ was found to be $7.471 \times 10^{-24} \mathrm{JK}^{-1}$ regardless of the number of $\mathrm{CuO} \mathrm{O}_{2}$ planes; though the specific heat per unit mass, Sommerfeld coefficient as well as entropy per unit mass decreased with an increase in the number of $\mathrm{CuO}_{2}$ planes. The entropy of a mole of Bismuth based cuprates at $\mathrm{T}_{\mathrm{C}}$ was found to be $5.603 \times 10^{-24} \mathrm{JK}^{-1}$ irrespective of the $\mathrm{T}_{\mathrm{C}}$ or mass. The peak Sommerfeld coefficient temperature was noted to occur at the ratio $\mathrm{T} / \mathrm{T}_{\mathrm{C}}=0.66$ in the bismuth based cuprates.
\end{abstract}

Keywords: Superconductivity, Sommerfeld Coefficient, Specific Heat, Entropy

\section{Introduction}

Cuprate superconductivity has been studied for the past three decades due to the foreseen applications that will revolutionize the world if the microscopic mechanism behind high temperature superconductivity is discovered. Superconductivity was first discovered by Kamerligh Onnes in 1911 [1], and a further discovery of High Temperature superconductivity (HTS) by Bednorz and Mueller in 1986 [2] inspired intensive research in this area of cuprate high temperature superconductivity resulting to the discovery of $\mathrm{Y}-\mathrm{Ba}-\mathrm{Cu}-\mathrm{O}$ [3], Bi-Sr-Ca-Cu-O [4], Tl-Ba-Ca-Cu-O [5] and $\mathrm{Hg}-\mathrm{Ba}-\mathrm{Ca}-\mathrm{Cu}-\mathrm{O}$ [6]. The highest achieved experimental critical temperature $\left(\mathrm{T}_{\mathrm{C}}\right)$ is $140 \mathrm{~K}$ in optimally oxygen doped mercury cuprates superconductor $\mathrm{HgBa}_{2} \mathrm{Ca}_{2} \mathrm{Cu}_{3} \mathrm{O}_{\mathrm{x}}$ at ambient pressure [7] and $156 \mathrm{~K}$ under $2.5 \times 10^{10} \mathrm{~Pa}$ pressures in the same substance [8]. Iron based HTS was discovered in 2008 [9], whereas in 2015 the highest experimental $\mathrm{T}_{\mathrm{C}}$ of $203 \mathrm{~K}$ under pressures of $200 \mathrm{GPa}$ was found in a non - cuprate Sulfur Hydride $\left(\mathrm{H}_{2} \mathrm{~S}\right)[10]$.

The discovery of Bismuth based superconductor was first done by Michel et al., in 1987 [11]. The $\mathrm{T}_{\mathrm{C}}$ for this bismuth based cuprates ranged between 7 and $22 \mathrm{~K}$ containing Bi-Sr-
$\mathrm{Cu}-\mathrm{O}$. This discovery was overshadowed by the nearly immediate discovery of $\mathrm{YBa}_{2} \mathrm{Cu}_{3} \mathrm{O}_{7-\delta}$ which achieved a $\mathrm{T}_{\mathrm{C}}$ of $93 \mathrm{~K}$ [3]. However in January 1988, Maeda et al., reported a new compound of bismuth based cuprates after adding calcium to the initial compound used by Michel et al., and achieving a $\mathrm{T}_{\mathrm{C}}$ of about $110 \mathrm{~K}$ [4]. This encouraged researcher in this area to focus on bismuth based compound because the material's $T_{C}$ was above liquid nitrogen boiling point, an indication that nitrogen can be used as a cryogenic material rather than the expensive mercury. Bismuth based HTS cuprate compounds can be described by the general formula $\mathrm{Bi}_{2} \mathrm{Sr}_{2} \mathrm{Ca}_{\mathrm{n}-1} \mathrm{Cu}_{\mathrm{n}} \mathrm{O}_{2 \mathrm{n}+4+\delta}(n=1,2$ and 3$)$ where $\mathrm{n}$ imply the number of $\mathrm{CuO}_{2}$ planes, which results to three bismuth superconducting cuprates $\mathrm{Bi}_{2} \mathrm{Sr}_{2} \mathrm{CuO}_{6+\mathrm{x}}$ (one $\mathrm{CuO}_{2}$ plane with $\mathrm{T}_{\mathrm{C}}=7-22 \mathrm{~K}$ ), $\mathrm{Bi}_{2} \mathrm{Sr}_{2} \mathrm{CaCu}_{2} \mathrm{O}_{8+\mathrm{x}}$ (two $\mathrm{CuO}_{2}$ planes with $\mathrm{T}_{\mathrm{C}}=85$ $\mathrm{K})$ and $\mathrm{Bi}_{2} \mathrm{Sr}_{2} \mathrm{Ca}_{2} \mathrm{Cu}_{3} \mathrm{O}_{10+\mathrm{x}}$ (three $\mathrm{CuO}_{2}$ planes with $\mathrm{T}_{\mathrm{C}}=110$ $\mathrm{K})$ abbreviated as $\mathrm{Bi} 2201, \mathrm{Bi} 2212$ and $\mathrm{Bi} 2223$ respectively [4]. The maximum $\mathrm{T}_{\mathrm{C}}$ increases with increasing number of $\mathrm{CuO}_{2}$ planes $[12,13,14]$. This gave rise to the expectation that $\mathrm{T}_{\mathrm{C}}$ may increase further when the structural cell has more $\mathrm{CuO}_{2}$ layers [15]. Superconductivity occurs predominantly in 
the $\mathrm{CuO}_{2}$ planes.Interlayer and intra-layer interactions in layered HTS Cuprates play an important role in the enhancement of $\mathrm{T}_{\mathrm{C}}[12,16,17]$, whereas $\mathrm{T}_{\mathrm{C}}$ has been found to be proportional to the number of $\mathrm{Cu}-\mathrm{O}$ layer in $\mathrm{Bi}-\mathrm{Sr}-\mathrm{Ca}-$ $\mathrm{Cu}-\mathrm{O}$ and $\mathrm{Hg}-\mathrm{Ba}-\mathrm{Ca}-\mathrm{Cu}-\mathrm{O}$ compounds $[13,20]$. Table 1 below shows the number of cuprates plane and the $\mathrm{T}_{\mathrm{C}}$ of Bismuth based HTS cuprates.

Table 1. Bismuth based cuprates phases, their $T_{C}$ and Number of $\mathrm{CuO}_{2}$ planes.

\begin{tabular}{llll}
\hline CuprateCompound & $\begin{array}{l}\text { Short hand } \\
\text { notation }\end{array}$ & $\begin{array}{l}\text { MaximumT } \\
(\mathbf{K})\end{array}$ & $\begin{array}{l}\text { No of Cuprates } \\
\text { planes }\end{array}$ \\
\hline $\mathrm{Bi}_{2} \mathrm{Sr}_{2} \mathrm{CuO}_{6}$ & $\mathrm{Bi} 2201$ & 20 & 1 \\
$\mathrm{Bi}_{2} \mathrm{Sr}_{2} \mathrm{CaCu}_{2} \mathrm{O}_{8}$ & $\mathrm{Bi} 2212$ & 95 & 2 \\
$\mathrm{Bi}_{2} \mathrm{Sr}_{2} \mathrm{Ca}_{2} \mathrm{Cu}_{3} \mathrm{O}_{10}$ & $\mathrm{Bi} 2223$ & 110 & 3 \\
\hline
\end{tabular}

The Bi-based HTSC are superior to the YBCO in respect of higher $\mathrm{T}_{\mathrm{C}}$. This class of superconductors (unlike YBCO) are resistant to water or humid atmosphere and have the advantage of compositional / oxygen stability, e.g. some of its superconducting phases do not gain or lose oxygen, when the material is annealed at $850^{\circ} \mathrm{C}$ [12]. Another advantage of the $\mathrm{BSCCO}$ materials relates to the fact that $\mathrm{BiO}$ layers being Van der Waal bonded, this material can be easily rolled. This property has been utilized successfully for tape-casting and its texturing. Furthermore, Bi2223 has been used in making superconducting tape magnet for maglev train [19] and wires for large-scale and high-current applications [20]. This magnet is very successful and a train using this magnet has been shown to achieve a speed of up to $500 \mathrm{~km} / \mathrm{h}$ [19]. However, it is generally agreed that Bi2212 samples have not reached the degree of purity and structural perfection obtained in YBCO [12]; hence a theoretical study is advised.

\section{Theoretical Formulation}

The order parameter of an interaction between Cooper pair and electron is given by a ket (1).

$$
|\Psi\rangle=\prod_{k, q=1}^{n}\left(u_{k}+v_{k} a_{k}^{\dagger} a_{-k}^{\dagger}\right) a_{q}^{\dagger}|0\rangle
$$

From (1), Cooper pair in momentum state $k$, comprises of two electrons creation operators in state $k$, i.e. spin up $a_{k}^{\dagger}$, and spin down $a_{-k}^{\dagger}$. The independent electron in an excited state $q$ is created by $a_{q}^{\dagger}$ in a vacuum $|0\rangle$. Note that $u_{k}$ is the probability of a vacuum state $|0\rangle$ in momentum state $k$ being unoccupied by the Cooper pair $a_{k}^{\dagger} a_{-k}^{\dagger}$ whereas, $v_{k}$ is the probability of a vacuum state $|0\rangle$ in momentum state $k$ being occupied by the Cooper pair $a_{k}^{\dagger} a_{-k}^{\dagger}$. The complex conjugate for the order parameter is shown by a bra in (2) below

$$
\langle\Psi|=\prod_{k, q=1}^{n}\langle 0| a_{q}\left(u_{k}{ }^{*}+v_{k}{ }^{*} a_{k} a_{-k}\right)
$$

The Hamiltonian for the interaction between Cooper pair and an electron based on Froehlich equation is given as

$$
\widehat{H}=\sum_{q} \epsilon_{q} a_{q}^{\dagger} a_{q}+\sum_{k} \epsilon_{k} a_{k}^{\dagger} a_{-k}^{\dagger} a_{-k} a_{k}
$$

$$
\begin{gathered}
+\sum_{k, q} V_{k, q} a_{q}^{\dagger} a_{q} a_{k}^{\dagger} a_{-k}^{\dagger}-\sum_{k, q} V_{k, q} a_{q}^{\dagger} a_{q} a_{-k} a_{k} \\
-\sum_{q, k} U_{k} a_{q}^{\dagger} a_{k}^{\dagger} a_{-k}^{\dagger} a_{-k} a_{k} a_{q}
\end{gathered}
$$

From (3), $\epsilon_{q}$ and $\epsilon_{k}$ are the kinetic energies for an electron and Cooper pair respectively. $V_{k, q}$ is the positive interaction potential between the electron and the Cooper pairs whereas $U_{k}$ is the negative Coulomb's potential between the electron and the Cooper pair. The average energy needed during the interaction is written as

$$
E_{k}=\langle\Psi|\widehat{H}| \Psi\rangle
$$

Inserting (1) and its conjugate (2) as well as (3) into (4) and obeying the anti-commutation rule, the ground state energy $E_{k}$ is determined.

The following are the conditions for determining specific heat $\left(C_{V}\right)$, Sommerfeld coefficient $(\gamma)$, entropy $(\mathrm{S})$ and critical temperature $\left(\mathrm{T}_{\mathrm{C}}\right)$ of the system

$$
\begin{gathered}
C_{V}=\frac{d E_{n}}{d T} \\
\gamma=\frac{C_{V}}{T} \\
\mathrm{~S}=\int C_{V} \frac{d T}{T} \\
\left(\frac{\partial C_{V}}{\partial T}\right)_{T=T_{C}}=0
\end{gathered}
$$

\section{Results and Discussion}

\subsection{Energy of the System}

From figure 1 (a), the energy of a mole of Bi2201, Bi2212 and $\mathrm{Bi} 2223$ is $0.747 \times 10^{-22} \mathrm{~J}, 3.548 \times 10^{-22} \mathrm{~J}$, and $4.109 \times 10^{-22} \mathrm{~J}$ respectively at the $\mathrm{T}_{\mathrm{C}}$. The energy per unit mass is found to be $0.05977 \mathrm{JKg}^{-1}, 0.2466 \mathrm{JKg}^{-1}$ and 0.2466 $\mathrm{JKg}^{-1}$ respectively at $\mathrm{T}_{\mathrm{C}}$ as shown in figure 1(b). The shape of the graph relating energy to temperature in figure 1 is half stretched sigmoid curves. This shape of curve was also observed by other researchers [13, 14, 21, 22, 23, 24, 25]. For the Bismuth based cuprates, a decrease in temperature results to a decrease in energy (figure 1).The effect of number of particles on the thermal properties of a heavy nuclei system showed that a decrease in temperature leads to a reduced particle interaction with a decrease in energy [26]. This concurs with observations in figures 1 , that a decrease in temperature results into a decrease in energy which effectively implies a reduction in particle interaction as a result of reduced temperature. Comparatively the energy at $\mathrm{T}=\mathrm{T}_{\mathrm{C}}$ for an electron - Cooper pair interaction for $\mathrm{Tl} 2201$, $\mathrm{T} 12212$ and $\mathrm{T} 12223$ is $3.548 \times 10^{-22} \mathrm{~J}, 3.922 \times 10^{-22} \mathrm{~J}$, and $4.669 \times 10^{-22} \mathrm{~J}$ respectively [13]; whereas the energy of interaction for an electron - Cooper pair at $\mathrm{T}=\mathrm{T}_{\mathrm{C}}$ is found to be $3.661 \times 10^{-22} \mathrm{~J}, 4.781 \times 10^{-22} \mathrm{~J}$, and $5.043 \times 10^{-22} \mathrm{~J}$ for $\mathrm{Hg} 1201$, $\mathrm{Hg} 1212$ and $\mathrm{Hg} 1223$ respectively [14]. The ARPES measurements on BSCCO indicate a $d$-wave energy gap with 
$\Delta_{0} \sim 30 \mathrm{meV}$ [27] and $\Delta_{0} \sim 27 \mathrm{meV}$ [28]. From the comparative results it is noted that the experimental technique applied during experimental measurement determines the likely

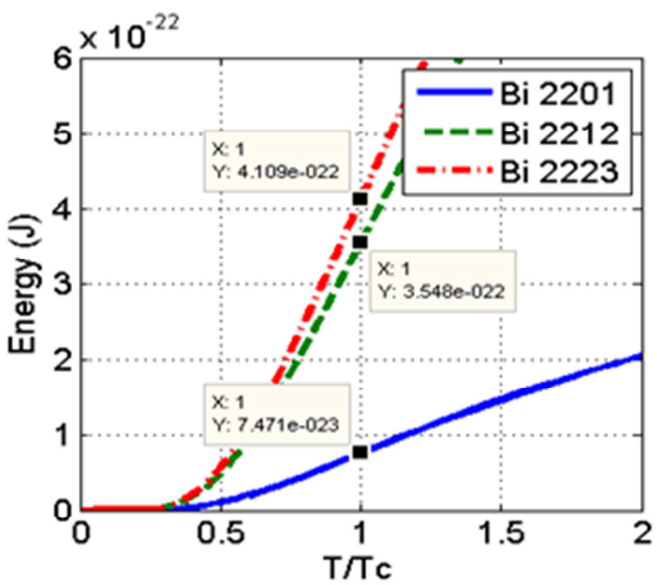

(a) energy of interaction and it is close to our prediction for Bismuth based cuprates.

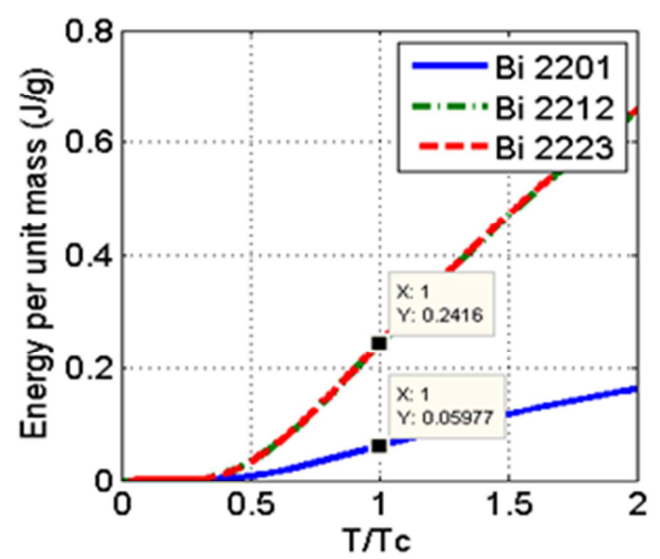

(b)

Figure 1. Energy of Bismuth based Cuprates as a function of Temperature (a) for a mole and (b) per unit mass.

\subsection{Specific Heat of the System}

The graph for specific heat as a function of $\mathrm{T} / \mathrm{T}_{\mathrm{C}}$ shown in figures 2, are skewed Gaussian shaped curves. This has been observed by other scientists for varied materials under varied conditions [29-31]. The specific heat in a mole of Bismuth based cuprates is found to be $7.471 \times 10^{-24} \mathrm{JK}^{-1}$ at the $T_{C}$ of $\mathrm{Bi} 2201, \mathrm{Bi} 2212$ and $\mathrm{Bi} 2223$ as shown in figure 2 (a). The specific heat per unit mass in Bismuth based cuprates is found to be $5.977 \mathrm{mJg}^{-1} \mathrm{~K}^{-1}, 5.064 \mathrm{mJg}^{-1} \mathrm{~K}^{-1}$ and $4.393 \mathrm{mJg}^{-1} \mathrm{~K}^{-1}$ for Bi2201, Bi2212 and Bi2223 as shown in figure 2 (b).Peak specific heat occurs at critical temperature [32].
Comparatively Kibe [22] while studying the pairing symmetry of the singlet and triplet pairing observed specific heat capacity of $4.8 \times 10^{-23} \mathrm{JK}^{-1}$ at $\mathrm{T}_{\mathrm{C}}$. It has been noted that at $\mathrm{T}=\mathrm{T}_{\mathrm{C}}$, the specific heat for $\mathrm{T} 12201, \mathrm{~T} 12212$ and $\mathrm{T} 12223$ is $5.337 \mathrm{mJg}^{-1} \mathrm{~K}^{-1}, 4.597 \mathrm{mJg}^{-1} \mathrm{~K}^{-1}$, and $4.038 \mathrm{mJg}^{-1} \mathrm{~K}^{-1}$ respectively [13] whereas $\mathrm{Hg} 1201, \mathrm{Hg} 1212$ and $\mathrm{Hg} 1223$ has specific heat per unit mass of $7.463 \mathrm{mJg}^{-1} \mathrm{~K}^{-1}, 5.839 \mathrm{mJg}^{-1} \mathrm{~K}^{-1}$, and $4.965 \mathrm{mJg}^{-1} \mathrm{~K}^{-1}$ respectively [14]. We notice that at the $\mathrm{T}_{\mathrm{C}}$ for Bismuth based cuprates just as in the case for Thallium and mercury based HTS, as the number of $\mathrm{CuO}_{2}$ planes increases, the specific heat decreases proportionally $[13,14]$.

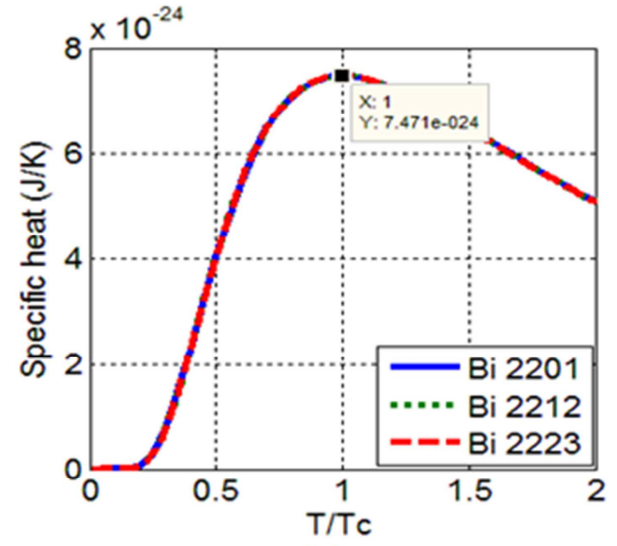

(a)

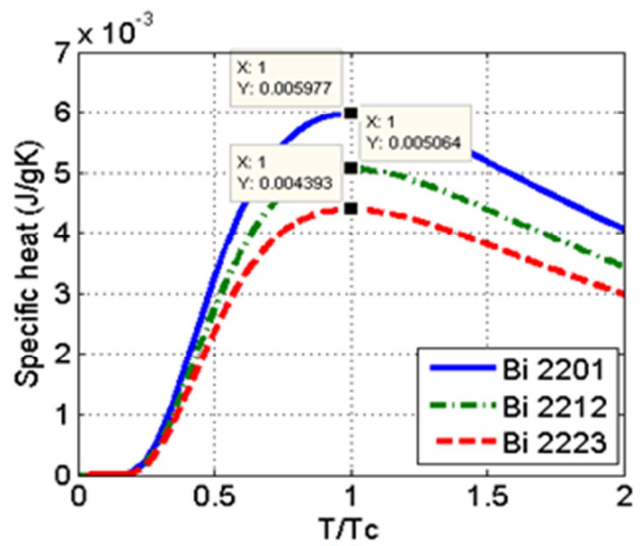

(b)

Figure 2. Specific heat for Bismuth based cuprates as a function of Temperature for (a) a mole of Bismuth based cuprates (b) a unit mass of bismuth based cuprates.

\subsection{Sommerfeld Coefficient of the System}

The Sommerfeld coefficient sometimes called electronic specific heat is a ratio of specific heat to the temperature. In the case of a mole of Bismuth based cuprates it is found to be $4.633 \times 10^{-25} \mathrm{~J} \mathrm{~K}^{-2}, 0.9763 \times 10^{-25} \mathrm{~J} \mathrm{~K}^{-2}$ and $0.8432 \times 10^{-25} \mathrm{JK}^{-2}$ at the $\mathrm{T}_{\mathrm{C}}$ of $\mathrm{Bi} 2201, \mathrm{Bi} 2212$ and $\mathrm{Bi} 2223$ respectively as shown in figure 3 (a). The Sommerfeld coefficient per unit mass in Bismuth based cuprates is found to be $7.413 \mathrm{mJg}^{-1} \mathrm{~K}^{-2}, 6.287 \mathrm{mJg}^{-1} \mathrm{~K}^{-2}$ and $5.454 \mathrm{mJg}^{-1} \mathrm{~K}^{-2}$ for $\mathrm{Bi} 2201, \mathrm{Bi} 2212$ and $\mathrm{Bi} 2223$ respectively as shown in figure 3 (b). 


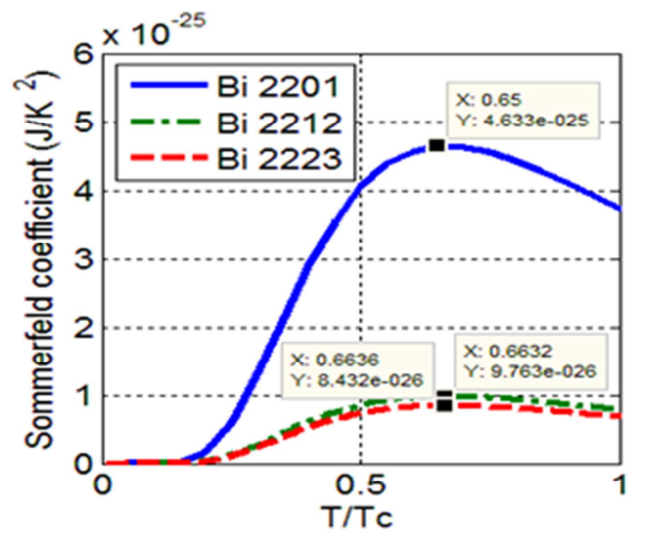

(a)

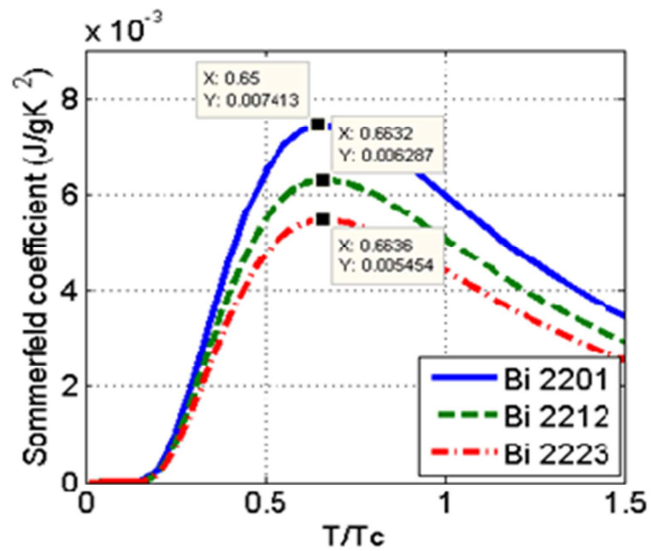

(b)

Figure 3. Sommerfeld coefficient as a function of temperature for Bismuth based cuprates in (a) a mole of BSCCO (b) a unit mass of BSCCO.

Comparatively the Sommerfeld coefficient for T12201, $\mathrm{T} 12212$ and $\mathrm{T} 12223$ is $6.975 \times 10^{-5} \mathrm{Jg}^{-1} \mathrm{~K}^{-2} ; 5.436 \times 10^{-5} \mathrm{Jg}^{-1} \mathrm{~K}^{-2}$; and $4.01 \times 10^{-5} \mathrm{Jg}^{-1} \mathrm{~K}^{-2}$ respectively [13]; whereas for $\mathrm{Hg} 1201$, $\mathrm{Hg} 1212$ and $\mathrm{Hg} 1223$ the Sommerfeld coefficient is $9.455 \times 10^{-5} \mathrm{Jg}^{-1} \mathrm{~K}^{-2} ; 5.664 \times 10^{-5} \mathrm{Jg}^{-1} \mathrm{~K}^{-2}$ and $4.567 \times 10^{-5} \mathrm{Jg}^{-1} \mathrm{~K}^{-2}$ respectively[14].The discrepancy between Sommerfeld coefficients arises from different extent of imperfections in samples of HTS cuprates used, as well as from inaccurate normalization that arises from imprecise oxygen composition determination [33, 34]. The structure of bismuth clip rates is very similar to the structure of thallium cuprates such as TI220I, T12212 and TI2223, with bismuth replaced by thallium, and strontium replaced by barium. In spite of similar structural features of bismuth and thallium compounds, there are differences in superconducting and normal-state properties [12]. From figure 3, the peak Sommerfeld coefficient occurs at a truncated temperature $\mathrm{T} / \mathrm{T}_{\mathrm{C}}=0.6$ for all Bismuth based cuprates. This has also been observed in mercury based cuprates [13], and thallium based cuprates [14].In conclusion, the number of planes of $\mathrm{CuO}_{2}$ is inversely proportional to theSommerfeld coefficient $[13,14]$.

\subsection{Entropy of the System}

Entropy is the disorder experienced in the material media.

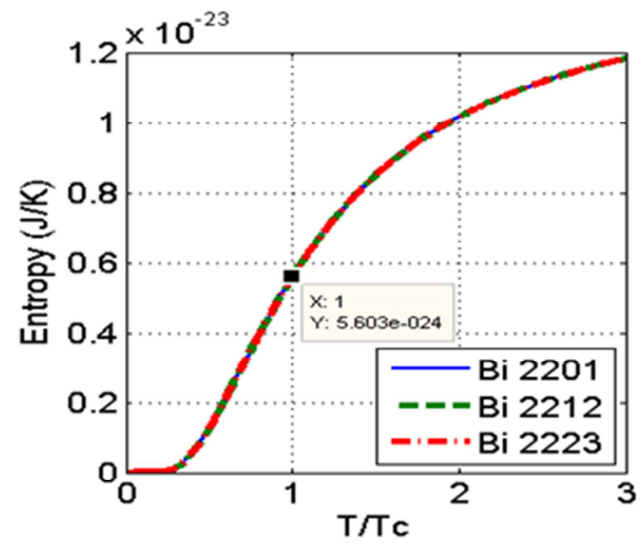

(a)
In case of a mole of Bismuth based cuprates is found to be $5.603 \times 10^{-24} \mathrm{JK}^{-1}$ at the $\mathrm{T}_{\mathrm{C}}$ of Bi2201, Bi2212 and Bi2223 as shown in figure 4 (a). Nearly similar entropy has been found per mole for: YBCO with value $3.036 \times 10^{-24}$ Junit cell $^{-1} \mathrm{~K}^{-1}$ [35]; whereas Rapando [23] based on theoretically study using the dipole mediated t-J model (t-J-d) found entropy to be $5.04693 \times 10^{-22} \mathrm{JK}^{-1}$. The specific heat per unit mass in Bismuth based cuprates is found to be $4.482 \mathrm{mJg}^{-1} \mathrm{~K}^{-1}, 3.798 \mathrm{mJg}^{-1} \mathrm{~K}^{-1}$ and 3,295 $\mathrm{mJg}^{-1} \mathrm{~K}^{-1}$ for Bi2201, Bi2212 and Bi2223 as shown in figure 4 (b).

When the temperature is lowered from a higher value to a lower value, the entropy also decreases and the HTS Cuprates material becomes more ordered. Other scientists have also made similar observation on the trend of entropy below $\mathrm{T}_{\mathrm{C}}[13,14,23,24]$. Comparatively, the entropy for T12201, T12212 and T12223 was found to be $4.003 \mathrm{mJg}^{-1} \mathrm{~K}^{-1}$, $3.448 \mathrm{mJg}^{-1} \mathrm{~K}^{-1}$ and $3.028 \mathrm{mJg}^{-1} \mathrm{~K}^{-1}$ respectively [13], while $\mathrm{Hg} 1201, \mathrm{Hg} 1212$ and $\mathrm{Hg} 1223 \mathrm{had}^{-1}$ entropy per unit mass of $5.597 \mathrm{mJg}^{-1} \mathrm{~K}^{-1}, \quad 4.38 \mathrm{mJg}^{-1} \mathrm{~K}^{-1}$ and $3.794 \mathrm{mJg}^{-1} \mathrm{~K}^{-1}$ respectively [14]. From the results, entropy decreases with an increasing number of $\mathrm{CuO}_{2}$ planes in bismuth based cuprates as observed in thallium based cuprates [13], and mercury based cuprates [14].

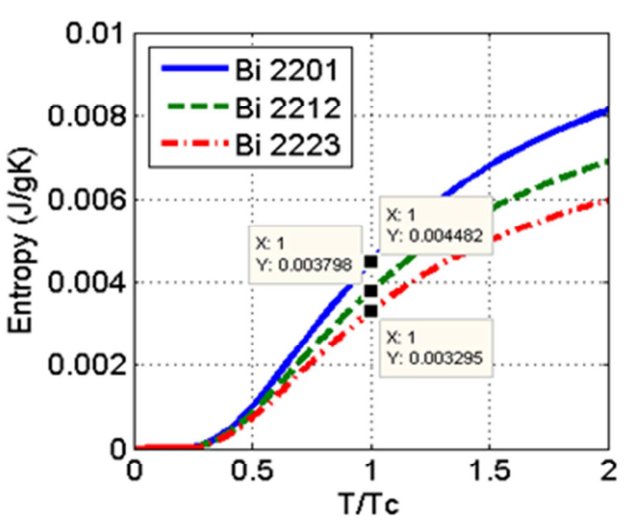

(b) 


\section{Conclusion}

In conclusion it'snoted that at $\mathrm{T}=\mathrm{T}_{\mathrm{C}}$ the energy of interaction in a three electron model in Bismuth based cupratesincreases with an increase in the number of $\mathrm{CuO}_{2}$ planes. It is noted that a decrease in the specific heat per unit mass results in a proportional increase in the number of $\mathrm{CuO}_{2}$ planes. Sommerfeld coefficient decreases with an increase in number of $\mathrm{CuO}_{2}$ planes, Specific heat and entropy per mole are constants not depending on $\mathrm{CuO}_{2}$ planes. When considered per unit mass entropy decreases with an increase in the number of $\mathrm{CuO}_{2}$ planes.

\section{References}

[1] Onnes H. K., (1911), The resistance of pure mercury at helium temperatures, Commun.,Phys. Lab. Univ. Leiden12:120.

[2] Bednorz G. J., and Mueller K. A., (1986), Possible high $T_{c}$ superconductivity in the $\mathrm{Ba}-\mathrm{La}-\mathrm{Cu}-\mathrm{O}$ system, Zeitschrift fur Physik B, 64 (1): 189-193.

[3] Wu M. K., Ashburn J. R., Torng C. J., Hor P. H., Meng R. L., Gao L., Huang Z. J., Wang Y. Q., and Chu C. W., (1987), Superconductivity at $93 \mathrm{~K}$ in a New Mixed-Phase $\mathrm{Y}-\mathrm{Ba}-\mathrm{Cu}-\mathrm{O}$ Compound System at Ambient Pressure, Physical Review Letters, 58 (9): 908-910.

[4] Maeda H, Tanaka Y, Fikutomi M, Asano T (1988). A New High-Tc Oxide Superconductor without a Rare Earth Element, Jpn. J. Appl. Phys. 27: L209- L210.

[5] Sheng Z. Z. and Hermann A. M. (1988), Superconductivity in the rare-earth free $\mathrm{Tl}-\mathrm{Ba}-\mathrm{Cu}-\mathrm{O}$ system above liquid nitrogen temperature. Nature,332: 55-58.

[6] Schilling A., Cantoni M., Guo J. D., Ott H. R., (1993), Superconductivity above $130 \mathrm{~K}$ in the $\mathrm{Hg}-\mathrm{Ba}-\mathrm{Ca}-\mathrm{Cu}-\mathrm{O}$ system, Nature, 363:56-58.

[7] Onbasil U., Ozdemir G. Z., and Asian O., (2009), Symmetry breaking and topological solitons in mercury based d-wave superconductivity, Chaos soliton and fractals,42(4):1980 1989.

[8] Ihara H., Hırobayashi M., Tanino H., Tokiwa K., Ozawa H., Akahana Y., Kawamura H., (1993), The Resistivity Measurements of $\mathrm{HgBa}_{2} \mathrm{Ca}_{2} \mathrm{Cu}_{3} \mathrm{O}_{8+\mathrm{x}}$ and $\mathrm{HgBa}_{2} \mathrm{Ca}_{3} \mathrm{Cu}_{4} \mathrm{O}_{10+x}$ Superconductors under High Pressure,Japan J. App. Physics, 32:L1732-L1734.

[9] Kamihara Y., Watanabe T., Hirano M., and Hosono H. (2008), Iron-Based Layered Superconductor LaO1-x FxFeAs (x = $0.05-0.12$ ) with $\mathrm{Tc}=26 \mathrm{~K}$. Journal of the American Chemical Society, 130(11):3296-3297.

[10] Drozdov A. P., Eremets M. I., Troyan I. A., Ksenofontov V. and Shylin S. I., (2015), Conventional superconductivity at 203 kelvin at high pressures in the sulfur hydride system, Nature, 525: 73-79.

[11] Michel C., Hervieu M., Borel M. M., Grandin A., Deslandes F., Provost J. and Raveau B. (1987), Superconductivity in the Bi-Sr-Cu-O system, Zeitschrift fur Physik B, 68(4): 421-423.

[12] Mourachkine A., (2002), High-Temperature Superconductivity in Cuprates: The Nonlinear Mechanism and Tunneling Measurements, Kluwer Academic Publishers, New York.

[13] Odhiambo J. O., Sakwa T. W., Rapando B.W. and Ayodo Y. $\mathrm{K}$., (2016), Effect of $\mathrm{CuO}_{2}$ plane on the thermodynamic properties of double Tl-O layered Cuprate based on an interaction between Cooper pair and an electron, International Journal of Physics and Mathematical Sciences, 6(2): 69-77.

[14] Odhiambo J. O., Sakwa T. W., Ayodo Y. K., and Rapando B.W., (2016), Thermodynamic properties of Mercury based cuprate due to Cooper pair - electron interaction, Journal of Multidisciplinary Engineering Science and Technology, 3(7): 5241-5248.

[15] Chen X. J. and Lin H. Q., (2004), Variation of the superconducting transition temperature of hole-doped copper oxides, Physics Review B, 69:104518.

[16] Sigei F. K., (2013), Theoretical determination of specific heat and critical temperature of High-Tccuprates superconductors based on intralayer and interlayer interactions. MSc (Physics) Thesis, University of Eldoret, Kenya.

[17] Tešanović Z., (1987), Role of interlayer coupling in oxide superconductors, Physics Review B, 36: 2364.

[18] Greenblatt M., Li S., McMills L. E. H. and Ramanujachary K. V., (1990), Chemistry and Superconductivity of ThalliumBased cuprates, Studies of High Temp Superconductors. U. S. Naval Research Technical Report, No. 56.

[19] Md. Atikur R., Md. Zahidur R., and Md. Nurush S., (2015), A Review on Cuprate Based Superconducting Materials Including Characteristics and Applications, American Journal of Physics and Applications, 3(2):39-56.

[20] Cyrot M. and Pavuna D., (1995), Introduction to Superconductivity and High-Tc Materials, World Scientific, Singapore.

[21] Ayodo Y. K., Khanna K. M., and Sakwa W. T., (2010), Thermodynamical variations and stability of a binary BoseFermi system, Indian Journal of Pure \& Applied Physics, 48: 886-892.

[22] Kibe E. H., (2015), Thermodynamic Properties of Heavy Fermion Superconductors, M.Sc (Physics) thesis, Masinde Muliro University of Science and Technology.

[23] Rapando B. W., Khanna K. M., Tonui J. K., Sakwa T. W., Muguro K. M.,Kibe H., Ayodo Y. K., and Sarai A., (2015), The dipole mediated t-J model forhigh-Tc superconductivity, International Journal of Physics and Mathematical Sciences, $5(3): 32-37$.

[24] Sakwa T. W., Ayodo Y. K., Sarai A., Khanna K. M., Rapando B. W., and Mukoya A. K., (2013), Thermodynamics of a Grand-Canonical Binary System atLow Temperatures; International Journal of Physics and Mathematical Sciences; 3(2):87-98.

[25] Waswa M. N., Ayodo Y. K., Sakwa T. W., Ndinya B. and Kibe, H (2017), Doped Mott Insulators within the Strong Coupling Regime, International Journal of Recent Engineering Research and Development, 2 (7): 102-108.

[26] Ndinya B. O., and Okello A., (2014), Thermodynamics properties of a system with finite heavy mass nuclei, American Journal of Modern Physics, 3(6): 240-244, ISSN: 2326-8867 (Print), ISSN: 2326-8891 (Online). 
[27] Norman M. R., Randeria M., Ding H., and Campuzano J. C. (1995), Phenomenological models for the gap anisotropy of $\mathrm{Bi}_{2} \mathrm{Sr}_{2} \mathrm{CaCu}_{2} \mathrm{O}_{8}$ as measured by Angle-resolved Photoemission Spectroscopy, Physical Review B., 52: 615.

[28] Ding H., Campuzano J. C., Bellman A. F., Yokoya T., Norman M. R., Randeria M., Takahashi T., Katayama-Yoshida H., Mochiku T., Kadowaki K., and Jennings G. (1995), Momentum Dependence of the Superconducting Gap in $\mathrm{Bi}_{2} \mathrm{Sr}_{2} \mathrm{CaCu}_{2} \mathrm{O}_{8}$. Physical Review Letters, 74: 2784-2787.

[29] Abdel-Hafiez M., Zhang Y., He Z., Zhao J., Bergmann C., Krellner C., Duan C.,Lu X., Luo H., Dai P., and Chen X., (2015), Nodeless superconductivity in the presence of spindensity wave in pnictide superconductors:The case of $\mathrm{BaFe}_{2-x} \mathrm{Ni}_{x} \mathrm{As}_{2} ;$ Physical Review B,91: 024510(1) 024510(10).

[30] Bagatskii M. I., Sumarokov V. V., Barabashko M. S., Dolbin A. V., and Sundqvist B., (2015), The low-temperature heat capacity of fullerite C60, Journal of Low Temperature Physics, 41(8):630-636.
[31] Bhattacharyya A., Adroja D., Kase N., Hillier A., Akimitsu J., and Strydom A., (2015),Unconventional superconductivity in $\mathrm{Y}_{5} \mathrm{Rh}_{6} \mathrm{Sn}_{18}$ probed by muon spin relaxation, Scientific Report,5:12926(1)-12926(8).

[32] Saxena K. A. (2010), High Temperature Superconductors, Springer-Verlag, Berlin.

[33] Bessergeven V. G., Kovalevskaya Y. A., Naumov V. N., and Frolova G. I., (1995), Phonon characteristic of $\mathrm{YBa}_{2} \mathrm{Cu}_{3} \mathrm{O}_{7-\delta}$, Physica C, 245:36-40.

[34] Royston L. N., (2001), Specific heat measurements on chevrel phase materials exhibiting coexistence of superconductivity and magnetism, Ph.D. Thesis, Physics department, Durham University, Online: http://etheses.dur.ac.uk/3849

[35] Loram J. W., Mirza K. A., Cooper J. R., and Liang W. Y., (1993), Electronic Specific heat of $\mathrm{YBa}_{2} \mathrm{Cu}_{3} \mathrm{O}_{6+\mathrm{x}}$ from 1.8 to 300K, Physics Review Letters, 71:1740-1743. 